\title{
Filigrane
}

Écoutes psychanalytiques

\section{"Mère ne vois-tu pas que je brûle ? " Esquisse d'une compréhension de la dynamique familiale des migrants clandestins disparus}

\section{Wael Garnaoui}

Volume 28, numéro 2, 2019

URI : https://id.erudit.org/iderudit/1069697ar

DOI : https://doi.org/10.7202/1069697ar

Aller au sommaire du numéro

Éditeur(s)

Santé mentale et société

ISSN

1192-1412 (imprimé)

1911-4656 (numérique)

Découvrir la revue

Citer cet article

Garnaoui, W. (2019). « Mère ne vois-tu pas que je brûle ? " Esquisse d'une compréhension de la dynamique familiale des migrants clandestins disparus. Filigrane, 28(2), 137-152. https://doi.org/10.7202/1069697ar
Résumé de l'article

Le texte propose une lecture psychanalytique du phénomène migratoire des harragas à partir de matériel recueilli auprès des mères de jeunes Tunisiens ayant risqué leur vie pour rejoindre l'Europe. Deux dimensions analytiques organisent notre propos. D’un côté, il s'agit de questionner les motifs inconscients qui structurent cette forme de migration en lien avec le désir maternel et la relation mère-fils plus généralement. De l'autre côté, nous revenons sur les conséquences psychiques désastreuses de l'acte migratoire clandestin (deuil collectif, traumatisme familial, etc.), des conséquences qui ne se limitent pas aux personnes migrantes, mais touchent également l'entourage familial et plus particulièrement les mères. 


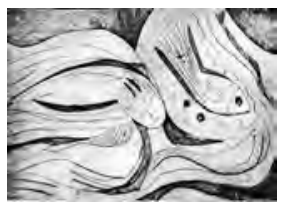

\title{
"Mère ne vois-tu pas que je brûle? " Esquisse d'une compréhension de la dynamique familiale des migrants clandestins disparus
}

\author{
Wael Garnaoui
}

Résumé: Le texte propose une lecture psychanalytique du phénomène migratoire des harragas à partir de matériel recueilli auprès des mères de jeunes Tunisiens ayant risqué leur vie pour rejoindre l'Europe. Deux dimensions analytiques organisent notre propos. D'un côté, il s'agit de questionner les motifs inconscients qui structurent cette forme de migration en lien avec le désir maternel et la relation mère-fils plus généralement. De l'autre côté, nous revenons sur les conséquences psychiques désastreuses de l'acte migratoire clandestin (deuil collectif, traumatisme familial, etc.), des conséquences qui ne se limitent pas aux personnes migrantes, mais touchent également l'entourage familial et plus particulièrement les mères.

Mots clés: Tunisie; migration clandestine; harraga; mère; disparus; psychanalyse; anthropologie.

\begin{abstract}
The text presents a psychoanalytic approach of the migratory phenomenon of harragas using data collected from the mothers of young Tunisians who risked their lives to reach Europe. Our paper follows two analytical paths. On the one hand, it attempts to identify the unconscious motives which constitute this form of migration together with the maternal desire and the general mother-son relationship. On the other hand, we return to the disastrous psychic consequences of the clandestine migratory act (collective mourning, family trauma, etc.). These consequences are not only limited to the migrants themselves but affect the whole family and especially mothers.
\end{abstract}

Key words: Tunisia; illegal migration; harraga; mother; psychoanalysis; anthropology. 


\section{Traverser la mer pour satisfaire la mère?}

Homme libre, toujours tu chériras la mer!

La mer est ton miroir, tu contemples ton âme

Dans le déroulement infini de sa lame,

Et ton esprit n'est pas un gouffre moins amer.

(Charles Baudelaire, L'homme et la mer)

$\mathrm{N}$ otre recherche ${ }^{1}$ est inspirée par l'actualité pressante et oppressante d'un phénomène social de plus en plus répandu: la migration des individus d'un pays à l'autre, d'un continent à l'autre, sous le signe d'une clandestinité totale.

Partant d'une problématique générale - celle qui porte sur le passage téméraire de nombreux Tunisiens vers l'Europe pour échapper à la pauvreté et y bâtir une vie meilleure -, il s'agit pour nous d'examiner la condition des mères des migrants clandestins pour comprendre à la fois l'impact de cette forme de migration sur la famille ainsi que les motifs inconscients qui semblent structurer ces départs et qui seraient liés à la condition familiale, et plus particulièrement aux relations mères-fils. Les deux questions se croisent et se superposent puisque les mères que nous avons rencontrées et les récits cliniques qui ressortent de ces rencontres font état des souffrances des mères, mais aussi d'une réflexivité permanente chez ces dernières quant aux conditions des départs, des disparitions et de la vie après la migration. Pour le dire autrement, les mères nous parlent de leurs souffrances, qu'elles lient à la situation familiale qui structure le départ. L'expérience psychique des mères en lien avec la migration du fils ne se forme pas uniquement à partir du moment du départ ou de la disparition : elle est en interaction avec l'expérience migratoire de l'enfant avant même le passage à l'acte migratoire. Cette expérience partage un univers migratoire et un discours sur la migration présent dans la famille avant le départ. Suivant notre hypothèse issue des rencontres avec les mères, cette expérience est souvent décisive dans ces départs. Nous restituerons ainsi ces deux dimensions problématiques révélées par les entretiens, à savoir les souffrances familiales incarnées par les mères et l'appartenance de ces dernières à l'ensemble de l'univers migratoire structurant la harga du fils. Une fois que l'on a épuisé les thèses qui explorent la causalité socio-économique et qui mettent le doigt sur des problèmes sociaux telle la migration clandestine (Noureddine, 2013), la question de l'investissement subjectif et psychique des migrants et des liens avec l'entourage proche demeure encore ouverte. Ce qui nous intéresse davantage ici est d'aller au-delà d'une évidence sociologique fonctionnaliste 
pour interroger les principales implications du désir maternel dans son lien possible à la décision du départ.

Rappelons d'abord quelques éléments de définition. Notre propos s'articule autour des harragas et de leurs mères. «Harragas» signifie littéralement «brûleurs de route». La harga, terme partagé par les peuples de l'ensemble du Maghreb, désigne l'acte de brûler les frontières pour se rendre en Europe, à la suite d'une traversée de la Méditerranée sur des embarcations de fortune. Le terme s'est généralisé ensuite pour désigner d'autres formes de migration clandestine, toujours vers l'Europe, et qui consistent par exemple à rester sur le territoire européen après l'expiration d'un visa ou d'un titre de séjour provisoire, à effectuer un voyage clandestin à bord d'un navire transportant des immigrés légitimes, etc. Souvent, les jeunes qui réussissent l'entrée dans l'espace européen «brûlent» leurs cartes d'identité afin d'éviter d'être identifiés par la police et expulsés vers leur pays d'origine. Le phénomène désigne donc à la fois l'acte de brûler littéralement les papiers du pays de provenance et le fait de «brûler» les frontières.

C'est à partir d'une pratique clinique et d'une recherche dans le champ de la psychanalyse (Garnaoui, 2013, p. 14) conduites auprès des familles des harragas que nous aborderons l'expérience des mères de migrants et de la traversée clandestine des migrants. La recherche porte sur six mères de jeunes clandestins, dont trois mères de «brûleurs» portés disparus et deux mères de brûleurs arrivés à bon port, la dernière étant la mère d'un «brûleur» arrêté par la police tunisienne au moment où il s'apprêtait à prendre la mer. Nous mobiliserons également dans ce texte notre travail d'accompagnement clinique réalisé entre 2014 et 2016 auprès de quinze familles de migrants clandestins. Il s'agissait d'un espace d'écoute, d'échanges d'expériences et de vécu, dans lequel il était question pour nous d'évaluer et d'identifier la souffrance psychologique des endeuillées dans ses différents aspects, pour pouvoir enfin assurer un soutien psychologique et un suivi thérapeutique. La prise en charge clinique s'est adressée aux familles, mais ce sont principalement les mères qui se sont mobilisées comme nous le montrerons plus tard. Les entretiens cliniques ont eu lieu dans le siège de l'association Psychologues solidaires à Tunis, mais aussi chez des familles habitant différentes régions de la Tunisie. La prise en charge clinique des quinze familles s'inscrit dans le projet Generazioni Perdute, Pensare la cura in territori di frontiera porté par l'association Frantz Fanon à Turin et l'association Psychologues solidaires à Tunis.

Les entretiens que nous avons eus à l'occasion de la prise en charge des mères de migrants disparus ont en effet attiré mon attention sur la 
singularité du fils harrag par rapport à ses frères, sa relation avec la mère étant différente de celle qu'entretient cette dernière avec le reste de la fratrie. De façon récurrente, il est le préféré de sa mère.

Dans nos recherches antérieures portant sur les motivations psychiques des départs, nous avons élaboré quelques traits caractérisant les candidats de la harga. Des traits communs se dégagent des profils variés des harraga: ils sont des jeunes hommes prêts à tout pour relever ce défi mortel et incarner ainsi l'enfant sauveur qui parviendra à tirer sa famille de la misère tout en déjouant la mort, la mer et la police. Dans le cadre du voisinage ainsi que dans le cadre familial, envoyer depuis l'étranger des sommes d'argent à sa famille confère un prestige certain à l'individu et lui assure une ascension sociale. Le harrag acquiert le statut de héros, dans son quartier, sa famille et aux yeux de sa mère en particulier: en l'aidant financièrement, le harrag comble son désir (Garnaoui, 2013, p. 14).

Plusieurs cas attestent chez ces jeunes hommes un désir d'une si forte reconnaissance narcissique qu'ils n'hésitent pas à réitérer cette expérience quand elle échoue. Le regard fixé sur l'Eldorado européen, ils seraient tout entiers captifs de leur obsession qui leur confère une identité nouvelle (on a pu évoquer à ce sujet une " altérité eldoradienne»). Chargés « de la douleur de l'Autre» (Lacan, 1956-1957), c'est-à-dire d'une mère dont le désir est vectorisé par un Ailleurs, l'impossibilité de satisfaire intégralement la demande de cet Autre les conduirait le plus souvent vers une issue fatale qu'ils endossent à leur propre compte, faute d'être en mesure d'en déchiffrer les causes profondes.

Ces jeunes qui, en «brûlant» les frontières maritimes, brûlent les interdits de la loi, mettent en acte une insubordination qui revêt tous les aspects d'une mise en péril «totale». En se jetant dans la mer/mère, ils réalisent à leur insu des fantasmes archaïques induits par la demande de l'Autre, qui, à la manière d'un impératif catégorique, surmoïque, les conduit à faire ce «choix», à la fois «forcé et féroce» (Pestre, 2015).

Songeons au tatouage que porte l'un des jeunes hommes dont j'ai interviewé la mère, qui nous montre la photo de lui qui reste après la migration: sur l'un de ses bras est gravé «mère», Limima, et sur l'autre Roma, le nom de la ville vers laquelle il souhaitait émigrer. Le corps devient récit, tente d'immortaliser des événements clés de l'existence. Il n'y a rien de plus archaïque que la relation à la mère, que le tatouage met en équivalence avec la relation à un ailleurs qui est Rome.

Il y a ces cas qui ressortent de l'analyse clinique et où le lien des fils brûleurs à leur mère est privilégié, au point de virer à la «symbiose mortifère». 
Nous rencontrons cette relation par exemple dans le cas de $\mathrm{M}^{\mathrm{me}} \mathrm{C}$. qui a mis en place un stratagème afin de dissuader son fils A. de «brûler». Pour elle, il s'agit d'amener ce dernier à faire un choix entre la harga et l'amour maternel, qu'elle symbolise par «Bzizla» (qui veut dire «le sein»). Elle s'exprime ainsi: «Il veut partir depuis longtemps, chaque fois qu'il veut partir, je lui dis: "Tu oublieras mon sein, si tu pars, je ne te le pardonnerai jamais." »

Or, comme tout symptôme, l'acte de brûler est à double face - ainsi qu'on le verra dans les entretiens suivants. Le désir de «brûler» ne va pas toujours et seulement à la rencontre du désir de l'Autre maternel, mais exprime aussi la nécessité vitale de s'en séparer.

Avant de commencer la restitution de notre étude à partir des familles, rappelons simplement le contexte de notre activité et de notre accompagnement clinique. En 2011, la Tunisie était à l'origine de nombreux exodes des harragas vers l'Europe. Les chiffres fournis par Frontex (l'Agence européenne de contrôle des frontières) «dénoncent » que, parmi 64261 migrants, 27864 Tunisiens avaient traversé le canal de Sicile par la mer pendant la seule année 2011 - une année, rappelons-le, qui a connu le déferlement de la Révolution tunisienne ainsi que de mouvements sociaux contestataires inédits dans l'ensemble des pays de la Méditerranée. C'est au milieu de ces transformations et crises politiques majeures que les chiffres des migrants clandestins ont explosé pour donner lieu à plus de 1000 Tunisiens morts et disparus en mer en 2012, selon le Forum tunisien des droits économiques et sociaux (FTDES, 2012).

\section{Le cas de $\mathbf{M}^{\mathrm{me}} \mathbf{N}{ }^{2}$}

$\mathrm{M}^{\mathrm{me}} \mathrm{N}$. habite dans une ville côtière de l'est de la Tunisie près de Monastir. Elle est mère de cinq garçons. Deux d'entre eux ont «brûlé» et ont réussi à atteindre la France. Le benjamin fut arrêté par la police pendant sa traversée clandestine. Aux dires de ses voisins, $\mathrm{M}^{\mathrm{me}} \mathrm{N}$. est une femme de fort caractère (ils étaient persuadés qu'elle n'accepterait pas de me rencontrer). Or, l'ayant trouvée devant sa maison, je me suis présenté à elle et, contre toute attente, elle a spontanément accepté d'échanger avec moi à propos de la harga de ses enfants. Elle me raconte que l'aîné n'arrivait pas à gagner sa vie, qu'il ne pouvait même pas subvenir à ses besoins élémentaires. «Il a tout perdu, tout gâché», poursuit-elle. Puis elle se remémore la période pendant laquelle elle n'avait pas de nouvelles de son fils qui avait traversé la mer. Mais plutôt que d'évoquer le souci que lui causait cette absence sans nouvelles, elle banalise l'acte de «brûler», employant à maintes reprises l'expression «labès», qui 
veut dire «tout va bien». Et quand elle se souvient devant moi du jour où son fils a pris le large, son récit est dépourvu d'affect, laconique, "plaqué» pour ainsi dire: «Un jour ses frères ont murmuré "el harga" - il a brûlé -, pour me dire qu'il avait émigré clandestinement.»

Du deuxième de ses fils, qui comme son aîné a fait l'expérience de la harga, $\mathrm{M}^{\mathrm{me}} \mathrm{N}$. dit qu'il a exprimé ouvertement son désir de partir. «Il était plus clair, il me l'a dit franchement. Je l'ai aidé avec le peu que j'ai. Enfin il a pu rejoindre son frère et, Dieu merci, les nouvelles qu'on a d'eux sont bonnes.» Après m'avoir dit que le troisième de ses enfants gagne bien sa vie, et qu'il compte demeurer dans son pays, elle évoque l'échec de la tentative de harga du benjamin. À ses yeux, la traversée de la mer est un acte qui nécessite du courage, certes, mais qui ne revêt pas de connotation «négative» ou «dangereuse », rien en tout cas qui aurait pu la retenir d'insuffler le désir de partir chez ses enfants en les aidant financièrement et moralement: «Il m'a dit qu'il a 1000 dinars, je lui ai donné davantage, Dieu l'a aidé, je l'ai aidé et il est parti.»

Un autre point, essentiel pour comprendre les causes de la harga, apparaît à la fin de notre entretien: $\mathrm{M}^{\mathrm{me}} \mathrm{N}$. emploie le terme «fesdin», qui veut dire «libéré» ou, plus exactement, tel que j'ai pu le saisir dans son discours, attaché à la civilisation moderne considérée comme déviée: délié de la tradition, le désir d'Occident devient infini. Elle ajoute: «Mes fils qui sont restés ici sont plus calmes que les autres, les autres sont libérés.»

\section{$\mathbf{M}^{\mathrm{me}} \mathbf{M}$., ou la tradition comme antidote à la transgression}

$\mathrm{M}^{\mathrm{me}} \mathrm{M}$., une veuve de 50 ans, est mère de trois garçons et d'une fille. Originaire d'une petite ville au nord de la Tunisie, elle vit dans une grande ville dans la région du Sahel. Son aîné, B., âgé de 30 ans, est forgeron et marié. Il a déjà tenté de quitter le pays, a débarqué clandestinement vers la France avec ses amis, mais a été arrêté par la police des frontières italienne.

Au début de l'entretien, la mère évoque l'affliction (ghasra) qu'elle et sa famille ont endurée pendant l'expérience de la harga. "Ghasra» signifie peine et douleur, mais rappelle aussi l'expérience de l'accouchement: événement intime, unique, central dans la vie. $\mathrm{M}^{\mathrm{me}} \mathrm{M}$. exprime également le regret que B. ne soit pas parvenu à accomplir sa harga - comprise par elle comme une nouvelle naissance? "On a eu une épreuve qui nous a beaucoup affligés: il a brûlé, mais n'est pas arrivé.»

Elle se souvient qu'elle a beaucoup supplié son époux d'accepter d'aider B. à partir, en arguant du fait qu'un de ses frères à elle, habitant en France, 
aiderait B. à s'y installer. Elle estimait en effet que «seuls se perdent ceux qui n'ont personne pour les secourir». Bien qu'il n'ait pas consenti sur le fond à ce projet, le père finit par céder en mettant à la disposition du jeune homme une somme d'argent épargnée en vue du mariage de sa sœur.

Une fois l'argent obtenu, B. partit avec son cousin et ses amis acheter «l'instrument» de ce passage. Cet «instrument», qu'il se gardait de nommer en toutes lettres, ils l'ont caché dans le garage d'un certain Sami, qui s'en est rendu compte et est allé déclarer sa découverte à la police. Nos harragas en puissance l'ont alors évacué du garage et se sont soustraits aux recherches de la police. $\mathrm{M}^{\mathrm{me}} \mathrm{M}$. dit qu'au moment de leur départ, « elle a senti son cœur cesser de battre». Elle et son mari étaient affolés au point d'en perdre le sommeil: «Nous ne pouvions dormir ni jour ni nuit.»

Nous avons ensuite demandé à $\mathrm{M}^{\mathrm{me}} \mathrm{M}$. de nous parler de la façon dont elle avait vécu l'expérience de la harga. Elle répond que c'était une attente angoissante. Elle a prié sans relâche pour que son fils revienne, tout en insistant sur le fait que la famille n'avait guère pu s'opposer à sa décision: "On prie Dieu jour et nuit pour que mon fils revienne, je n'ai besoin ni de la France ni de rien, on prie Dieu jour et nuit, mais on ne peut rien faire.»

Lorsque nous lui demandons comment l'idée de la harga est venue à son fils, elle explique que tout a commencé le jour où B. a inauguré son atelier, un projet que quelqu'un «lui avait mis dans la tête».

La contradiction devient patente: bien qu'ayant soutenu l'idée que quelqu'un avait mis le projet dans la tête de B., elle affirme à présent «qu'elle ne voulait pas afficher devant son époux son désir d'aider B. à partir», tout en persistant à penser qu'il «allait trouver son bonheur et sa vie à l'étranger».

Dans une autre perspective, $\mathrm{M}^{\mathrm{me}} \mathrm{M}$. établit une distinction intéressante entre les trois frères. Le frère cadet de B., explique-t-elle, n'irait jamais outremer, de peur de mettre sa vie en péril. Il n'aurait pas même l'idée de quitter le pays en avion. Quant au plus jeune, présent lors de notre entretien, la mère n'a jamais abordé ce sujet avec lui. Elle revient enfin à la question de son aîné, pour souligner que son plan de harga a failli la rendre folle. Il ne cessait de se rabaisser et de dénigrer la Tunisie, lui rappelant à tout moment que son travail à l'atelier était harassant et ne lui rapportait quasiment pas un sou: "Il n'avait qu'une idée en tête, partir à l'étranger, dehors.»

En revanche, après qu'il eut été sauvé de la noyade, B. s'est mis à prétendre qu'il préférait rendre l'âme que de revivre cet épisode. À ce stade de notre discussion, la mère semble revoir la pâleur cadavérique de son fils 
arraché du ventre de la mer. Elle reprend son souffle et me dévoile, tout en remerciant Dieu, que le revoir au milieu de ses amis avait été un moment inoubliable.

Revenu à son quartier, B. travaille quelque temps sans faire allusion à sa harga, jusqu'à ce que son vieux fantasme le reprenne.

«Je lui dis: attends, un peu de patience; il se calme pendant deux jours et, le troisième, il est repris par son idée fixe.»

S'inclinant une nouvelle fois, sa famille avait pris en charge les documents administratifs de son atelier et son dossier d'émigration. Elle avait tout prévu, sauf un compte bancaire au consulat. Nouvelle déception, donnant à $\mathrm{M}^{\mathrm{me}} \mathrm{M}$. à le sentiment qu'elle a de nouveau failli perdre son fils.

Bien que la loi tunisienne criminalise ce type d'immigration, B. n'a pas cessé de vouloir transgresser cette loi. Le harrag est tout autant celui qui "grille» un visa en séjournant à l'étranger au-delà des délais autorisés, que celui qui passe par la mer sans visa. Au début des années 1990, les harragas se cachaient plutôt dans les cales des bateaux de lignes régulières. Mais quand il est devenu de plus en plus risqué de se glisser sur les bateaux en raison du durcissement des contrôles, le brûleur a opté pour un nouveau stratagème: sur les 1300 kilomètres de côtes tunisiennes, les candidats à l'émigration ont recouru aux services de professionnels du passage ou se sont auto-organisés en achetant des zodiacs, des moteurs ou encore des GPS en vue de départs collectifs, avec une complexité d'organisation et un coût financier hors de portée pour les plus démunis.

Afin de sauver son fils, $\mathrm{M}^{\mathrm{me}} \mathrm{M}$. a penché pour un mariage. Était-ce la solution? Il semble que oui. Devenu responsable de la famille après le décès de son père, il était «maintenant devenu un homme». $\mathrm{M}^{\mathrm{me}} \mathrm{M}$. précise:

— Pendant cette période il était perdu, donc je l'ai marié.

- Et qu'est-il devenu, après le mariage?

- Il a épousé la fille et il a totalement changé, il est devenu un vrai homme, responsable de sa maison, de sa femme et de ses futurs enfants.

Le mariage et la harga sont deux expériences dont les filigranes s'entretissent: tous les deux sont porteurs d'un trait virilisant.

Les paroles de cette mère nous font comprendre que la décision prise par B. a été influencée par sa souffrance, comme si le risque qu'il avait pris était un acte réparateur pour sa mère. C'est une réponse à l'Autre maternel. Son projet réalisait à la fois le désir d'un accès au monde des hommes et la 
satisfaction des désirs d'une mère - le désir de sa mère de le savoir en Europe était parfaitement clair.

À travers sa harga, B. convoque une réponse de l'Autre. Elle lui sert d'étiquette en le nommant et le faisant exister en tant qu'Homme. En fait, il y a une équivalence entre ses actes et lui. Le désir émerge instantanément et s'extériorise en éclat.

Le passage par la mer fait impact, «troue» et laisse une trace. Il s'agit de la trace du sujet qu'il cherche à être, un marquage du territoire qui le définit dans son existence. Il est tout entier dans ce que lui renvoie l'effet de son agir à l'extérieur de lui. L'agir le fait surgir dans le regard des autres, c'est un acte qui fait être: «Il est l'objet convoité du désir de l'Autre.» (Texier, 2011)

Cette histoire de «brûlage» peut être envisagée comme une sorte de version locale (géographiquement et historiquement) du drame œdipien: les harragas seraient des œdipes, qui à la fois cherchent à combler leur mère et à s'en débarrasser (ils leur offrent le bouquet de fleurs de l'Eldorado occidental et simultanément se font la belle, se tirent de chez elles, les "plaquent»). Leur désir de satisfaire leur mère se transformerait en un désir de transgression des frontières et de reconnaissance sociale et politique. Interrogeant la relation particulière entre mère et enfant au sein des sociétés musulmanes maghrébines, Abdallah Laroui écrit:

L'homme est toujours dans les bras de sa mère, parle son dialecte, caractérisé par ses qualités, réalise ses objectifs, fait tout pour la satisfaire, vit sous sa protection et ne dépasse pas l'horizon de sa vie, même lorsqu'il migre et s'éloigne d'elle. (Laroui, 2009, 146-147; nous traduisons)

Selon lui, une vraie modernisation devrait passer par un «sevrage» de la culture maternelle, au fondement de la tradition musulmane. Rappelons simplement, dans cette perspective, que «modernité» ne signifie pas chez ce penseur une simple reprise essentialiste du processus civilisationnel occidental. Il entend l'accès à une technicité rationnelle et bureaucratique ainsi qu'une démocratisation directe et radicale qui permet davantage d'autonomie aux individus. Quant à nous, nous analysons la figure de la mère qui configure le désir du fils (celui qui transgresse la loi pour satisfaire le désir puissant de la mère) comme s'il s'agit du lieu de la confrontation entre deux 
mondes: le monde de la modernité et celui de la tradition. Bien entendu, il n'y a là aucun jugement moral en jeu. Mieux encore, nous voyons dans le traditionalisme configuré par le pouvoir des femmes une force de résistance (au sens politique) contre la technique rationnelle et mercantile absolue (ceci s'illustre simplement par la volonté de transgresser la loi des frontières des États-nations modernes). Cette résistance peut prendre des chemins tragiques comme révélé dans notre étude de cas où la harga débouche sur la mort. Bouhdiba, en s'inspirant des Mille et une nuits, a parlé du mythe de Jawdar qui, selon lui, est un profil type d'un œedipe local maghrébin, voire arabo-musulman: il est «fort légitime de voir dans le complexe de Jawdar la forme, spécifique à la culture arabo-musulmane, du complexe d'EEdipe» (Bouhdiba, 1978). Le mythe de Jawdar met en question une relation particulière de l'enfant à la mère marquée par la fusion corporelle et par une dépendance qui peut durer toute la vie (Ghorbal, 1981).

Ce point de vue qui met l'accent sur la liaison politico-existentielle entre la mère et le fils paraît pertinent au regard de l'histoire de $\mathrm{M}^{\mathrm{me}} \mathrm{M}$. et de son fils B., car elle dévoile la faillite d'un mode de vie ancien, d'une culture, ou du moins des transformations rapides qui bouleversent les structures sociales traditionnelles, les croyances et les rapports sociaux dans les sociétés maghrébines en général. On voit du reste que les pères n’ont plus voix au chapitre, phénomène probablement lié à la désactivation de la «fonction paternelle» (Hurstel, 2004), mais aussi à la réduction de la femme à son rôle de mère: «Tout commence et tout finit dans la mère. D'où ce double combat de la femme arabe musulmane par rapport à l'homme et par rapport à la nature. Rien d'étonnant qu'elle soit épuisée par ce double effort et qu'elle ait payé très cher la maintenance de la personnalité arabo-musulmane» (Bouhdiba, 1975). Encore récemment, l'ailleurs était situé non loin, dans le village voisin ou dans la grande ville proche. Aujourd'hui, il a basculé de l'autre côté de la mer. Ce phénomène est général, mais ce qui donne aux mouvements migratoires une dimension dramatique, c'est le simple fait, à la fois incroyable et inacceptable, qu'aux peuples de certaines parties du monde (le Maghreb en particulier, alors qu'il est près de l'Europe à la fois historiquement et économiquement) soit refusé le droit de circuler librement sur la surface de la planète. L'Ici n'étant plus attractif, l'Ailleurs étant interdit, reste la transgression, ou la régression (emprunter un schéma de mariage traditionnel où les mères jouent un rôle décisif dans le choix de l'épouse, retour à des interprétations religieuses radicales, etc.). La figure contemporaine du migrant clandestin constitue un exemple paradigmatique d'une 
clinique du déplacement à l'heure de la globalisation, elle relève l'intrication de la pulsionnalité avec la scène politique et sociale (Pestre, 2015, p. 15-20).

Nous avons montré dans cette première partie d'analyse que l'acte de la migration anime chez les mères un penchant d'idéalisation des fils migrants qui est tout à fait singulier et qui transforme radicalement le rapport qu'elles ont avec le monde. Certaines paroles des femmes rencontrées pourraient, certes, être comprises comme des signes d'un travail de deuil (idéalisation de la personne perdue), comme ceci est montré par Freud qui introduit la notion de deuil comme étant «la réaction à la perte d'une personne aimée ou d'une abstraction mise à sa place» (Freud, 1915). Or, l'idéalisation n'est pas intrinsèque à la mort et à la disparition comme nous l'avons souligné à travers les cas présentés. Pour vérifier cette hypothèse, nous avons choisi de rencontrer des mères qui ont vécu l'expérience de la migration du fils sans que cet acte débouche sur la disparition ou la mort de ce dernier. En revanche, la disparition du fils migrant dédouble l'investissement pulsionnel des mères dans le processus, comme nous allons le montrer dans la partie qui suit.

\section{Perte et reconstitution dans le contexte de la révolution}

La disparition d'un être cher dans un contexte flou entraîne des blessures psychiques évidentes. Et la rumination de la disparition, après-coup, est encore plus traumatisante. Car quoi qu'il en soit du sort du disparu, celui que suppose l'imaginaire anxieux est toujours plus cruel. Selon Freud, «le traumatisme est un débordement psychique par excès d'excitation. L'effraction traumatique est assez forte pour anéantir les pare-excitations.» (Freud, 1920) Et la disparition sous sa forme la plus violente est une effraction traumatique. Ce que Lacan appelle le «trou-matisme» (Lacan, 1974) figure l'ouverture d'un gouffre dans lequel risque de tomber l'endeuillé, ou au bord duquel il tente de garder l'équilibre.

Chaque semaine, pendant deux ans, des mères de harragas se sont réunies dans une de leurs maisons ou dans le local de l'association qu'elles ont créée (Terre pour tous) pour débattre des moyens à mettre en œuvre pour retrouver leurs fils disparus. Elles se sont également regroupées sur l'artère principale de Tunis, l'avenue Habib-Bourguiba, pour (ré) inventer de nouveaux symboles et ritualités les préservant d'un effondrement psychique - des manifestations qui font penser aux regroupements des mères de la place de Mai en Argentine, réclamant que la lumière soit faite sur la disparition de leurs enfants. La vérité est leur premier souci: «S’ils sont morts nous 
voulons leurs cadavres», car pour elles «la mort est plus simple à gérer que la disparition» (Garnaoui, 2013).

Entre-temps, trois des mères de Tunisiens disparus ont fait une tentative de suicide, dont $\mathrm{M}^{\mathrm{me}} \mathrm{J}$., mère de $\mathrm{N}$., disparu depuis mars 2011, qui a tenté de s'immoler par le feu en avril 2012 devant sa maison après que le "passeur» de son fils lui eut dit: «Va faire le deuil de ton fils.» Tout se passe comme si «l'acting-out» des jeunes harragas tunisiens et la mise en scène de leur devenir-hommes était passé de harga au sens d' «immigration clandestine» à harga au sens d' "immolation ». À plusieurs reprises lors de nos entretiens, des mères de harragas nous disent: «Je veux mourir pour éteindre le feu qui brûle dans mon cœur.» On songe ici à Mohammed Bouazizi, «l'homme calciné» (Benslama, 2013), qui s'est immolé par le feu dans la foulée d'une ultime humiliation: une gifle administrée par une femme policière, allumant la mèche de la révolution tunisienne.

Nous avons retrouvé chez plusieurs membres des familles des disparus une symptomatologie qui ressemble beaucoup aux états de stress post-traumatique, avec syndrome de reviviscence et d'évitement comme:

- Évitement de toute situation susceptible de rappeler la mer et la mort par noyade: aller à la plage, voir la mer (même à la télévision), manger du poisson...

- Chez certains, la seule vue d'un poisson est susceptible de déclencher une attaque de panique. D'autres évitent même de prendre un bain et ne supportent plus l'idée de plonger la tête sous l'eau.

- Évitement de toute situation ou événement susceptible de rappeler l'absence du disparu comme les réunions de famille, les dîners en famille et les jours de fêtes telles que l'a'id ou le ramadan.

- La rumination anxieuse alimentée par une culpabilité consciente est pire que toute situation réelle de reviviscence. Car la première explosion traumatique, consécutive à la disparition, cède la place à un tableau imaginaire. L'esprit de l'endeuillé devient le lieu même de la disparition, le théâtre cruel d'une perte inlassablement figurée.

Pas de corps à enterrer, pas de deuil. En revanche, est-il possible d'enterrer un spectre? Sans l'épreuve de réalité, c'est-à-dire sans la certitude de la mort, le fantôme du disparu hante l'esprit de ses proches. L'endeuillé luimême devient le lieu du non-repos de la personne disparue. Ceux qui ont rencontré les familles des disparus savent bien ce dont il s'agit: des corps fatigués, des visages abîmés, des yeux ternis et, parfois, des cadavres ambulants. 


\section{Traumatisme dans la famille}

Sur les quinze personnes que nous avons pu suivre durant notre prise en charge, nous n'avons rencontré que deux pères. Cela ne veut pas dire que les autres ne souffraient pas de la disparition, mais plutôt qu'ils évitaient d'en parler ou d'être confrontés à ce qui pourrait réactiver le traumatisme de la perte.

Nous avons su aussi à travers les mères que certains pères culpabilisaient beaucoup en s'auto-accusant d'être responsables de la disparition du fils. D'autres accusaient leurs épouses et leur faisaient porter toute la responsabilité.

Les mères, en revanche, paraissaient plus concernées par la disparition, au point, comme déjà mentionné, de tenter de se suicider dans les trois mois qui ont suivi la disparition de leur fils. Pratiquement toutes les mères présentent une symptomatologie anxio-dépressive, associée parfois à des complications somatiques. Nous avons aussi remarqué que ces mères avaient une relation fusionnelle avec le fils disparu, ce qui donnait à la perte une dimension narcissique. Cette relation fusionnelle avec le fils fait parfois de celui-ci un rival du père, ce qui pourrait expliquer la grande culpabilité que nous avons retrouvée chez certains pères. La disparition du fils serait alors la réalisation d'un infanticide inconscient.

Penser le lien fraternel dans la prise en charge des familles endeuillées nous est apparu aussi comme une chose indispensable. Bien que nous ayons suivi seulement deux membres des fratries des disparus, nous avons constaté que la disparition d'un frère était d'un impact violent sur l'espace fraternel et sur le devenir de la fratrie. Il y a le deuil, le chagrin à l'égard de celui qui est disparu, mais également le deuil à l'égard de chacun des membres de la famille transformée par cette perte, qui ne sera plus jamais ce parent, ce frère ou cette sœur «d'avant».

Une des conséquences désastreuses de la disparition consistait dans le désinvestissement de la mère, surtout à l'égard du reste de la fratrie. $M^{\text {me }} R$. lançait d'une manière très crue des vœux de morts à l'égard de son fils «restant». En colère contre ce dernier, elle lui avoue à maintes reprises qu'elle aurait aimé le perdre à la place de son frère.

$\mathrm{M}^{\mathrm{me}} \mathrm{O}$., une autre mère, avait interdit à sa fille de se fiancer: aucune fête, aucune joie n'est possible sans le retour du disparu.

Nous avons retrouvé chez ces frères et sœurs doublement agressés (par la perte et l'empêchement) une forme de culpabilité du survivant qui serait à l'origine de cette même haine inconsciente qu'ils déploient à l'égard du frère 
disparu. Le petit-fils de $\mathrm{M}^{\mathrm{me}} \mathrm{S}$., âgé de 18 ans, a développé une conduite toxicomaniaque avec fugues récurrentes et baisse des performances scolaires. Sa mère rapporte qu'il ne veut plus rentrer à la maison et qu'il s'est installé chez un copain (un voisin du même quartier) pour éviter d'être confronté à son effondrement et son irritabilité. Elle a rapporté aussi que dans un premier temps (avant les fugues), son petit-fils a essayé de compenser la perte du grand frère en l'imitant et en s'identifiant à ce dernier.

Il est donc évident que la disparition peut mettre à mal l'espace fraternel et mettre en souffrance ce lien jusqu'à le rendre destructeur. Les répercussions vont au-delà de l'organisation intrapsychique et viennent contaminer l'espace intersubjectif du lien fraternel, qui se trouve profondément désorganisé.

Dans notre approche clinique, nous avons toujours considéré trois dimensions essentielles: la souffrance individuelle de chacun, les souffrances interpersonnelles et la famille tout entière, comme «organisme vivant» atteint au plus profond de son être.

\section{Résilience familiale}

Les familles des disparus ont essayé de se «résilier» à la perte en se reconstituant autour d'elle, et cela avant même notre intervention auprès d'elles. Le mot «résilience» désigne un processus psychique proche de ce que Freud appelait «sublimation» et désignerait la capacité de transformer en richesses intérieures les conséquences pénibles d'un traumatisme (De Mijolla, 2006). En effet, la liberté d'expression fraîchement acquise et l'élan contestataire post-révolution ont permis une organisation au sein d'associations. Le sort des disparus est devenu pour la première fois une cause défendable et susceptible de faire pression sur les gouvernements de l'aprèsrévolution. Faute de réparation psychologique ou matérielle, les familles des disparus cherchaient à obtenir une reconnaissance symbolique, à l'instar des familles des martyrs de la révolution.

Après tout, leurs disparus sont les victimes de la marginalisation économique et politique d'une partie du monde à l'heure de la globalisation. Dans cette quête de reconnaissance et de vérité sur les disparus, certains ont pu rencontrer des responsables, des ministres et des gens très haut placés.

Selon Aymen Daboussi, dans la Tunisie de l'après-révolution la perte est devenue un capital symbolique, un objet d'une valeur inestimable - ce qu'il identifie comme un «narcissisme suprême de la personne endeuillée $e^{3}$ ». Lors d'une manifestation pour la vérité sur les disparus, l'une des mères a 
tenté de percer une haie de sécurité. Un membre du service d'ordre haut gradé s'est mis devant elle pour l'empêcher de pénétrer dans l'enceinte du ministère. Furieux contre son acharnement, il lui crie «Tu ne sais pas qui je suis?» À son étonnement, elle répond: «Et toi, tu ne sais pas qui je suis?» «Et qui tu es?» À ce moment elle rétorque: «Je suis une mère mutilée». $\mathrm{M}^{\mathrm{me}} \mathrm{R}$. racontait avec fierté comment l'officier, les yeux remplis de larmes, s'est penché sur elle pour l'embrasser sur le front. La douleur face à l'absence des corps nécessite également d'être pensée par les autorités.

Le désir formulé par la mère à l'égard du fils qui se voit propulsé dans une dynamique psychoaffective (le désir de l'autre, et le devoir de devenir cet autre rêvé et fantasmé) se nourrit de politiques subjectives diverses. Dans ces politiques se croisent dominations coloniales, mensonge migratoire, hégémonie occidentale, fermetures de frontières, etc. La responsabilité plurielle est rarement identifiée. Seuls les parents et les enfants sont responsabilisés dans les devenirs tragiques qui se manifestent dans la mort et la disparition. Nous avons essayé de montrer combien le désir de transgresser les contraintes frontalières pourrait trouver racine dans le désir maternel. Bien sûr, ce dernier pourrait aussi être intriqué dans de nombreuses politiques productrices des subjectivités qui ne sont ni reconnues ni résolues.

Tous ces disparus, toutes ces pertes, tous ces sacrifices ne peuvent rester sans sens et ne peuvent être inutiles et ressentis comme "sans raison». Ils doivent avoir une place dans l'histoire de ces familles et même dans la mémoire collective des Tunisiens. Rester dans l'incertitude est insupportable. Notre rôle en tant que psychologues est de faire en sorte que ces familles trouvent à qui parler, qu'elles disposent d'une «adresse» où faire entendre le «trou-matisme» dont elles souffrent, et se donner ainsi une chance de réintégrer ce "trou» au tissu de leur histoire, de celle de leur pays et de celle des hommes - marquée par l'avènement d'une époque de la disparition.

Wael Garnaoui garnaoui-wael@live.fr 


\section{Notes}

1. Cet article reprend, en les synthétisant, deux communications scientifiques (celle de mon collègue Aymen Daboussi et la mienne) présentées, le 18 décembre 2015, lors d'un séminaire à l'Université de Tunis intitulé «La clinique de la migration. Penser le soin entre les deux rives de la Méditerranée». Ce séminaire prenait lui-même place dans le cadre d'un projet de prise en charge des familles des Harragas disparus dirigé par l'association Psychologues solidaires, créée dans la foulée de la révolution tunisienne.

2. Les cas restitués dans ce texte bénéficient des consentements des familles rencontrées. Nous anonymisons les noms et les lieux pour assurer la protection des données collectées. Les rencontres avec ces familles s'inscrivent dans un travail de recherche qui a eu lieu au sein de l'UFR d'études psychanalytiques de l'Université Paris 7 Diderot.

3. Conférence de restitution des cas cliniques étudiés: "Clinique de la migration. Penser le soin entre les deux rives de la Méditerranée», Université de Tunis (FSHST), 18 décembre 2015.

\section{Références}

Benslama, F. (2013). Soudain la révolution! De la Tunisie au monde arabe: la signification d’un soulèvement. Paris: Denoël.

Bouhdiba, A. (1975). La sexualité en islam. Paris: Presses universitaires de France.

Bouhdiba, A. (1978). L'enfant et la mère dans la société arabo-musulmane. Dans Culture et société. Tunis: Publications de l'Université de Tunis.

Freud, S. (1920). Au-delà du principe de plaisir. Dans CEuvres complètes, vol. XV (p. 273338). Paris: Presses universitaires de France.

Freud, S. (1986). Métapsychologie. Paris: Gallimard.

FTDES (2012). Les Tunisiens disparus en mer en 2012. Rapport du Forum tunisien pour les droits économiques et sociaux. Repéré à http://ftdes.net/rapports/disparusenmer2012. pdf

Garnaoui, W. (2013-2014). Migration clandestine «Harga». Entre désir et fantasme (Mémoire de maîtrise inédit). Université Paris Diderot.

Ghorbal, M. (1981). La personnalité maghrébine: noyau arabo-islamique, sociogenèse, psychogenèse. L'information psychiatrique, 57 (4), 441-449.

Hurstel, F. (2004). Penser la paternité contemporaine, raisonner sur la clinique. Dans Fonctions maternelle et paternelle (p. 87-100). Toulouse: Éditions Érès.

Lacan, J. (1994). Le séminaire. Livre IV (1956-1957): La relation d'objet. Paris: Le Seuil.

Lacan, J. (1974). Le séminaire. Livre XXI: Les non-dupes errent. Paris: Le Seuil.

Laroui, A. (2009). Min diwan al-siyasa. Casablanca: Al-Markaz al-thaqafi l -» arabi.

De Mijolla-Mellor, S. (2006). Sublimation et résilience. Dans B. Cyrulnik et P. Duval (dir.), Psychanalyse et résilience. Paris: Odile Jacob.

Noureddine, K. (2013). Adolescents harragas: risquer sa vie comme seule possibilité de réalisation de soi. Adolescence, 31 (3), 699-709. doi: 10,391 7/ado.085.0699

Pestre, É. (2015). Déplacements et subjectivités dans le monde globalisé. Quand le «migrant clandestin » brûle ses attaches premières. L'information psychiatrique, 91 (1) 15-20.

Tassel,F.(2013).Tunisie: disparusentreparadisetenfer.Extraits d'entretien avecWaelGarnaoui. Libération. Repéré à https://www.liberation.fr/planete/2013/09/13/tunisie-disparusentre-paradis-et-enfer_931783

Texier, D. (2011). Adolescences contemporaines. Toulouse: Éditions Érès. 